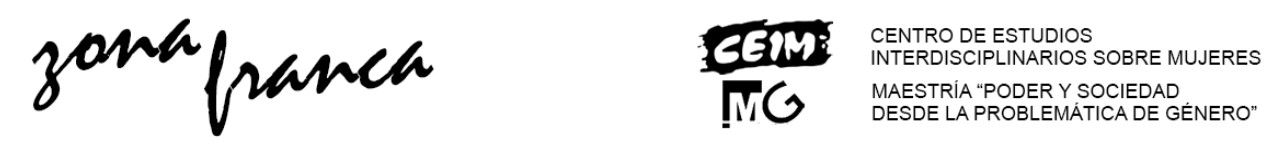

\title{
¿Hay alguien ahí? Simone de Beauvoir entre "yo" y "nosotras"
}

\author{
Mabel Alicia Campagnoli
}

\section{Resumen}

A setenta años de la edición de El segundo sexo nos interesa rescatar el valor político feminista de la escritura a través de la pluma de Simone de Beauvoir sin restringirla a sus aspectos teóricos sino desde una perspectiva transversal a los géneros discursivos que produjo (ensayo, novela, diario, autobiografía). Nuestra lectura busca apreciar la figura de Simone de Beauvoir como filósofa con perfil propio, a diferencia de las que solo la reconocen como escritora en un sentido literario menor, o en todo caso, como apéndice filosófico de las conceptualizaciones de Jean-Paul Sartre y de Maurice Merleau-Ponty.

\section{Palabras clave: Beauvoir - Discurso - Política - Feminismo \\ Anyone there? Simone de Beauvoir between "I" and "We"}

\section{Abstract}

Seventy years after the publication of The Second Sex we are interested in rescuing the feminist political value of writing through the pen of Simone de Beauvoir without restricting it to its philosophical aspects but from a transversal perspective to the discursive genres that produced (essay, novel, diary, autobiography). Our reading seeks to appreciate the figure of Simone de Beauvoir as a philosopher with her own profile, unlike those who only recognize her as a writer in a minor literary sense, or in any case, as a philosophical appendix of the conceptualizations of Jean-Paul Sartre and Maurice Merleau-Ponty.

Postdoctora en Estudios de Género (Universidad de Ciencias Empresariales y Sociales, Buenos Aires, Argentina). Doctora en Filosofía (Universidad Pablo de Olavide, Sevilla, España). Magíster en Análisis del Discurso (Universidad de Buenos Aires). Contacto: mabelcampagnoli@yahoo.com.ar

Campagnoli, Mabel Alicia. “¿Hay alguien ahí? Simone de Beauvoir entre "yo" y "nosotras” en Zona Franca. Revista del Centro de estudios Interdisciplinario sobre las Mujeres, y de la Maestría poder y sociedad desde la problemática de Género, №27, 2019 pp.87-102. ISSN, 2545-6504 Recibido: 18 de mayo 2019; Aceptado: 10 de abril 2019

Revista Zona Franca- Centro de estudios interdisciplinario sobre las mujeres (CEIM)- Maestría poder y sociedad desde la problemática de género (MG), Rosario, Argentina. ISSN, 2545-6504 http://zonafranca.unr.edu.ar/index.php/ZonaFranca| Numero 27 (2019). 
Key words: Beauvoir - Speech - Politics - Feminism

\section{Introducción}

Las reflexiones presentadas en este trabajo se iniciaron a partir de la invitación que me hiciera la colega Vera Wacksman a compartir una "Tarde de autobiografías" como actividad del proyecto a su cargo Autobiografías filosóficas. Problemas teóricos y estudios de caso (2017-2018) radicado en el Centro de Investigaciones en Filosofía (CleFi) de la Facultad de Humanidades y Ciencias de la Educación (FaHCE-UNLP). Agradezco a Vera la posibilidad de ese encuentro el 7 de noviembre de 2018, pues despertó en mí el interés por rescatar la escritura de Simone de Beauvoir como acción política. Como el entusiasmo continuó, me atrevo ahora a ofrecer aquí mi recorrido, a modo de homenaje, con motivo de los setenta años de la publicación de Le deuxième sexe.

\section{¿Hay alguien ahí?}

Judith Butler, en el prefacio a la segunda edición de El género en disputa. El feminismo y la subversión de la identidad afirma "El hecho de que pueda escribir de un modo autobiográfico no cambia, según yo, el sitio que ocupo como el sujeto que soy, aunque tal vez sí dé al lector cierto sentido de solaz al saber que hay alguien ahí" (Butler, 2001: 17; el énfasis es mío).

Dentro de las tradiciones filosóficas no es frecuente encontrar tal solaz. Aquí nos preguntamos si sucede en la escritura de Simone de Beauvoir. Principalmente hay dos motivos para sospecharlo, su adscripción al existencialismo y la fenomenología, por un lado; su posterior militancia feminista, por otro. Sin soslayar además, la práctica de una escritura de la intimidad que atraviesa toda su biografía, desde los Cahiers de jeunesse, 1926-1930 aún no editados en español, hasta La ceremonia del adiós en 1981, pasando por Diario de guerra, septiembre de 1939 - enero de 1941 y los cuatro tomos de su autobiografía (Memorias de una

Revista Zona Franca- Centro de estudios interdisciplinario sobre las mujeres (CEIM)- Maestría poder y sociedad desde la problemática de género (MG), Rosario, Argentina. ISSN, 2545-6504 http://zonafranca.unr.edu.ar/index.php/ZonaFranca| Numero 27 (2019). 
joven formal, 1958; La plenitud de la vida, 1960; La fuerza de las cosas, 1963; Final de cuentas, 1972).

Preguntarnos si hay alguien ahí excede un análisis lingüístico ya que ponemos en juego la recepción de la autora; es decir, una perspectiva que atiende no solo a las tecnologías de la escritura (Ong, 1984) sino también a las de la lectura (Bourdieu y Chartier, 2011). En consecuencia, nuestras consideraciones no dejan de ser una reflexión singular como ejercicio de conocimiento situado.

En tal sentido, nuestros comentarios no se basan en un recorrido exhaustivo de la obra de Beauvoir, sino en algunas enunciaciones del yo, en diferentes géneros literarios de su producción: ensayo, novela, diario, autobiografía... Igualmente, nos interesa observar la articulación con la primera persona del plural; en especial, con el desafío político que propone la autora al convocar la producción de un "nosotras" en su célebre ensayo El segundo sexo de 1949.

De esta manera, nuestra lectura busca apreciar la figura de Simone de Beauvoir como filósofa con perfil propio, a diferencia de las lecturas que solo la reconocen como escritora en un sentido literario menor, o en todo caso, como apéndice filosófico de las conceptualizaciones de Jean-Paul Sartre y de Maurice Merleau-Ponty.

Sobre la calificación de escritora, hay basamento en sus propias afirmaciones para sostenerla ("Sartre es un filósofo, yo no") y se ha discurrido mucho acerca de las motivaciones. Aquí seguimos la lectura que la considera filósofa, en línea con la perspectiva de Alejandra Ciriza: "yo pienso que ella es filósofa, yo pienso que si la filosofía no ha tenido "biografías" ha sido precisamente por esta suerte de juego a través del cual los filósofos se han ocupado de pronunciar su palabra pretendiendo que lo hacían por fuera de las condiciones bajo las cuales las pronunciaban; es decir, haciendo un juego de ocultamiento por el cual desmarcaban esas palabras de las marcas de lugar, de tiempo, de clase, de los compromisos éticos y políticos que la mayor parte de los filósofos y las

Revista Zona Franca- Centro de estudios interdisciplinario sobre las mujeres (CEIM)- Maestría poder y sociedad desde la problemática de género (MG), Rosario, Argentina. ISSN, 2545-6504 http://zonafranca.unr.edu.ar/index.php/ZonaFrancal Numero 27 (2019). 
filósofas han tenido y tienen. También pasa que para nosotras es muchísimo más difícil reconstruir nuestras propias genealogías precisamente por eso, porque los escritos filosóficos de las mujeres han sido considerados no filosóficos. Aun cuando Mary Wollstonecraft merece un lugar en la historia de la filosofía, ha sido considerada una polígrafa del siglo XIX, fines del siglo XVIII, una escritora más que contribuyó a la numerosa producción de escritura intrascendente de esa época. También tenemos el caso de las cartas filosóficas que no han sido consideradas como parte de la historia de la filosofía; por ejemplo, cuando una piensa en los intercambios filosóficos que la mayor parte de los filósofos varones no casualmente entablaron con mujeres, esos intercambios filosóficos no son considerados como filosofía, sino como una suerte de enseñanza que el maestro, siempre varón, tenía para dispensar a las siempre, eternas, aprendizas mujeres. Yo creo que las cosas son muchísimo más complejas. Cualquiera que haya dado clases alguna vez en su vida se da cuenta que, en realidad, aprende muchísimo del intercambio, que en realidad lo maravilloso de ser docente es que una enseña/aprende. La mayor parte de las veces las ideas más interesantes se nos ocurren en situaciones de intercambio con otras y con otros; entonces, esas mujeres consideradas no filósofas, simples aprendizas, eran en realidad una fuente de energía, de saber, de reflexión" (Ciriza en Grau y Castillo, 2012: 237238).

\section{Autoficción y espacio biográfico}

Desde que los estudios culturales cruzaron posestructuralismo con pragmatismo y produjeron el quiebre de la jerarquía entre saberes, todo relato es ficción; ni las ciencias, ni la historia, ni las biografías, tienen una relación privilegiada con la verdad. No hay más ontología del sujeto que la que provee el lenguaje. Este giro hacia la narrativa le otorga un valor especial al descentramiento de la voz enunciativa con pretensión de unicidad -teórica, filosófica- en beneficio de una pluralidad de puntos de vista (Rorty, 1990: 84).

Revista Zona Franca- Centro de estudios interdisciplinario sobre las mujeres (CEIM)- Maestría poder y sociedad desde la problemática de género (MG), Rosario, Argentina. ISSN, 2545-6504 http://zonafranca.unr.edu.ar/index.php/ZonaFranca| Numero 27 (2019). 
Así, la auto-biografía deviene auto-ficción y cuestiona la idea de "pacto autobiográfico" con la que Philippe Léjeune la definía al responder la pregunta ¿cómo decir la verdad sobre una/o misma/o? La solución que ofrecía Léjeune era el pacto autobiográfico, una forma de contrato entre autor/a y lector/a, por el cual el/la autobiógrafo/a no se compromete a una exactitud histórica sino al esfuerzo sincero por entender su existencia y su personalidad. Pero el supuesto de un sujeto pre-discursivo y la analogía con la autoridad jurídica implícita en la noción de "pacto", vuelve conflictivo el concepto que será sustituido por el de auto-ficción.

Como señala Paul Ricoeur, vivimos en lo narrativo, esto nos constituye a la vez que, paradójicamente, implica ficcionalidad: "Cuando leo un texto que contiene el término yo, lo encuentro ya disociado por la escritura de su escritor, aunque este escritor, en tanto locutor, haya sido un yo anclado; pero el hecho de estar escrito y no dicho, lo deja desanclado" (Ricoeur, 1990: 78). Es decir, la narración de una/o misma/o no es más que una ilusión, puesto que el/la autor/a pretende realizar lo imposible: relatar la historia de una primera persona que sólo existe en el presente de su enunciación.

En consecuencia, la ficción del yo en la enunciación resulta una instancia de constitución de subjetividad en el lenguaje. Así lo expresa July Chaneton para caracterizar la autoficción: "Cuando la narración es producida por el propio sujeto de enunciación en su discurso autorreferencial, se ha llamado "autoficcional", es decir, una narración de sí que se constituye inevitablemente por fuera del orden de lo verídico y que por lo tanto resulta en "la imposible narración de sí mismo". Dicha "imposibilidad", paradójicamente, no deja de ser altamente productiva en términos sociales. Efectos de sentido en relación con la configuración subjetiva resultante que son a la vez idiosincráticos -porque permiten al "yo" del enunciado adelantar una diferencia- y colectivos -porque provienen de y contribuyen a la producción de subjetividades sociales-." (Chaneton, 2007: 94).

Revista Zona Franca- Centro de estudios interdisciplinario sobre las mujeres (CEIM)- Maestría poder y sociedad desde la problemática de género (MG), Rosario, Argentina. ISSN, 2545-6504 http://zonafranca.unr.edu.ar/index.php/ZonaFrancal Numero 27 (2019). 
Nos interesa esta tensión entre lo individual y lo colectivo que señala Chaneton para aplicar su mirada a la lectura de textos de Simone de Beauvoir. Tensión que juega en la dimensión política de los pronombres, aunque ésta suele reconocerse solo en el "nosotros".

Para ello, articulamos a su vez la noción de autoficción con la de espacio biográfico, elaborada por Leonor Arfuch: "el espacio, como configuración mayor que el género [discursivo], permite una lectura analítica transversal, atenta a las modulaciones de una trama interdiscursiva que tiene un papel cada vez más preponderante en la construcción de la subjetividad contemporánea" (Arfuch, 2002: 102). Es la lectura que planteamos de transversalidad entre ensayo, novela y testimonio, donde buscaremos la tensión entre lo individual y lo colectivo.

\section{El cuerpo vivido}

¿Qué características tiene esta tensión en la autora, si cuando se entrega a la tarea de desencializar el "ser mujer" en El Segundo Sexo, la articulación entre el yo y el nosotras queda atrapada en el "cuerpo vivido"? Al respecto cabe considerar con Alejandra Ciriza que "en ese momento entre 1946 y 1949, [Simone de Beauvoir se hallaba] en una situación solitaria. Es precisamente por esto que su posición es profundamente ambivalente: a la vez que desea conquistar el mundo masculino hasta anexárselo, por decirlo de alguna manera, sabe que en el corazón de ese mundo está la clave de la subordinación de las mujeres. ¿Cómo escapar de esa situación? La estrategia que ella se plantea,

(...) es la estrategia del privilegio, es decir, yo soy una sujeto de dos mundos, puedo hablar en tanto procedente del mundo de las mujeres, pero al mismo tiempo he sido formada y educada en el mundo de los varones: soy una persona bilingüe, hablo las dos lenguas" (Ciriza, 2012: 15).

Ahora bien, estas dos lenguas, al referir al cuerpo vivido anulan en principio la posibilidad de relatarlo por fuera del androcentrismo. De ahí que, como señala

Revista Zona Franca- Centro de estudios interdisciplinario sobre las mujeres (CEIM)- Maestría poder y sociedad desde la problemática de género (MG), Rosario, Argentina. ISSN, 2545-6504 http://zonafranca.unr.edu.ar/index.php/ZonaFrancal Numero 27 (2019). 
María Carmen López Sáenz, "a diferencia de Maurice Merleau-Ponty que dice "yo soy mi cuerpo", Beauvoir diga que el cuerpo de la mujer es algo otro para ella. No lo vive como suyo; sólo es cuerpo para otros, para los hombres sin ser para-sí" (López Sáenz, 2012: 191).

En efecto, la mujer queda identificada como pura presencia ante el logos dominante masculino:

"La mujer tiene ovarios y un útero, y estas condiciones singulares la encierran en su subjetividad. De ella se dice gustosamente que piensa con las glándulas. El hombre olvida en su soberbia, que su anatomía también supone hormonas, testículos. Toma a su cuerpo como una relación directa y normal con el mundo, al cual cree aprehender en su objetividad, mientras que considera que el cuerpo de la mujer se encuentra como entorpecido por cuanto lo especifica" (Beauvoir, 1954: 14).

En este sentido, el cuerpo vivido que describe Beauvoir es la marca de la ambivalencia que constituye la situación de las mujeres a punto tal, que la modalidad de escritura se vuelve impersonal, retaceando tanto el yo como el nosotras:

"Esa ambivalencia se señala por el modo en que la mujer capta su cuerpo. Es un fardo: la especie lo roe, sangra todos los meses, prolifera pasivamente y no es para ella el puro instrumento de su aprehensión del mundo, sino una presencia opaca. No se asegura con certeza el placer, y le crea dolores que la desgarran; en cierra amenazas determinadas y se siente en peligro en su interior" (Beauvoir, 1954: 457).

De esta manera, la escritura en tercera persona del singular, "la mujer", muestra la tarea existencialista de caracterizar la condición del existente femenino, la existencia que llega al mundo sexuada como hembra. Si bien es la experiencia propia de la autora, la reflexión brinda la posibilidad de inteligir dicha experiencia a partir de condiciones de existencia que son comunes y que justamente condicionan la experiencia vivida. A punto tal la condicionan, que Beauvoir se va a

Revista Zona Franca- Centro de estudios interdisciplinario sobre las mujeres (CEIM)- Maestría poder y sociedad desde la problemática de género (MG), Rosario, Argentina. ISSN, 2545-6504 http://zonafranca.unr.edu.ar/index.php/ZonaFrancal Numero 27 (2019). 
distanciar de las perspectivas de Sartre y de Merleau-Ponty, al considerar que para muchas mujeres, su condición femenina constituye una opresión y no una mera situación (López Pardina, 1999) (Herrera, 2008). Tal es la importancia que alcanza el cuerpo vivido en su explicación, pues es «la condición» que permite comprender la opresión de las mujeres (López Sáenz, 2012).

\title{
Experiencias narrativas
}

Simone de Beauvoir reflexiona sobre la escritura de El segundo sexo en un volumen de su autobiografía, La fuerza de las cosas:

\begin{abstract}
“¿Qué había significado para mí ser mujer? Nunca había tenido sentimientos de inferioridad. Mi feminidad no me había molestado en nada. Para mí, le dije a Sartre, eso no había sido nunca un problema.

-Pero, de todas maneras, Castor, tú no has sido criada de la misma manera que un niño. Deberías analizarlo mejor...

Así lo hice y tuve una revelación: ese mundo era un mundo masculino. Mi infancia había sido alimentada por mitos forjados por hombres y yo no había actuado ante ellos de la misma manera que lo habría hecho si hubiese sido varón. Esto me interesó tanto que abandoné todo para ocuparme de la cuestión femenina en su totalidad" (Beauvoir, 1964: 92).
\end{abstract}

Vemos entonces que el inicio de la caracterización de la situación de las mujeres fue una reflexión situada en sí misma, un punto de partida en la situación propia, que también se evidencia al comienzo del ensayo, en la Introducción: "¿Qué es una mujer? El enunciado mismo del problema me sugiere inmediatamente una primera respuesta. Es significativo que yo lo plantee. A un hombre no se le hubiese ocurrido escribir un libro acerca de la situación singular que ocupan los machos en la humanidad. Si quiero definirme, me veo obligada a decir, en primer lugar; "Soy una mujer". Esta verdad constituye el fondo sobre el cual se yergue toda otra afirmación" (Beauvoir, 1954: 13).

Revista Zona Franca- Centro de estudios interdisciplinario sobre las mujeres (CEIM)- Maestría poder y sociedad desde la problemática de género (MG), Rosario, Argentina. ISSN, 2545-6504 http://zonafranca.unr.edu.ar/index.php/ZonaFranca| Numero 27 (2019). 
Por eso Mayra Leciñana Blanchard considera que aquí la afirmación "soy una mujer" funciona como dispositivo de enunciación que dispara la posibilidad del texto, cuyo punto central es el impersonal "No se nace mujer, se llega a serlo". Con ello, el proyecto de "relato personal" queda postergado hasta el inicio de publicación de su obra autobiográfica. La intérprete propone que "ese vaivén nos induce a tomar como eje el acto de la escritura de la primera persona para ver cómo narra ella el "llegar a ser” una mujer” (Leciñana, 2010: 94).

En este sentido, la invitación es a observar usos del "yo" de modo transversal, a lo largo del espacio biográfico, para rastrear el propósito que Beauvoir enuncia en otro tomo de su autobiografía, Memorias de una joven formal: "no quería hablar con esa vos abstracta que al oírla no me conmovía (...) soñaba escribir una "novela de la vida interior"; quería comunicar mi experiencia" (Beauvoir, 1999: 211).

Beauvoir bucea en su pasado hasta encontrar la escena que funda míticamente un comienzo. A los quince años dice haber escrito en el álbum de una amiga: "Había resuelto desde algún tiempo consagrar mi vida a tareas intelectuales (...) escribiendo una obra alimentada por mi historia me crearía yo misma de nuevo y justificaría mi existencia" (Ibíd: 145).

Allí mismo alude a los diarios de juventud, a los que retoma y también corrige: "me analizaba y me felicitaba de mi transformación. ¿En qué consistía exactamente? Mi diario lo explica mal; pasaba muchas horas en silencio y me faltaba perspectiva. Sin embargo al releerlo, algunas cosas me saltaron a la vista" (Ibíd: 192). Leciñana analiza este gesto como una manera de construirse: "Después de todo se trata de Memorias de una joven..., una postulación en tercera persona, marca del proyecto de sí como otra, de un atreverse a construirse" (Leciñana, 2010: 97). Así se juega en la escritura la noción existencialista de proyecto como marca de trascendencia en tanto implica un devenir transformativo.

Revista Zona Franca- Centro de estudios interdisciplinario sobre las mujeres (CEIM)- Maestría poder y sociedad desde la problemática de género (MG), Rosario, Argentina. ISSN, 2545-6504 http://zonafranca.unr.edu.ar/index.php/ZonaFrancal Numero 27 (2019). 
Ahora bien, la enunciación del "yo" también ocurre en una novela como $\mathrm{La}$ mujer rota elaborada en el formato de un diario íntimo que la protagonista comienza cuando su matrimonio está por naufragar. No podríamos tomarlo como auto-ficción pero sí podemos entenderlo como inscripción textual de un "yo" femenino en la exploración de sentidos de "llegar a ser mujer". En nuestra interpretación, las ficciones del yo en la escritura pueden considerarse como tecnologías del sí misma, en tanto productoras de una subjetividad sexuada.

En consecuencia, la mujer rota, que se adentra en un vacío existencial al descubrirse en la inmanencia del ser para otros, se evanece en la desesperación pero se afirma en la pluma que le permite aferrar la escritura: "No sé nada. No solamente quién soy sino cómo habría que ser (...) el mundo es un magma y no tengo ya contornos. ¿Cómo vivir sin creer en nada ni en mí misma?" (Beauvoir, 1975:33). Leciñana interpreta que esta mujer rota se escribe y desmonta una "situación", se desmarca, hasta que lo que queda es puro futuro, por delante un porvenir: "no se construye a sí misma. En un sentido un poco libre "se desconstruye" y no es a través de la conciencia ni de una voluntad radical, sino a través de una experiencia narrativa" (Leciñana, 2010: 99).

\section{Filosofía y feminismo}

...cuando se es mujer y filósofa es útil ser feminista para comprender lo que nos pasa (Le Dœuff, 1993: 51)

La escritora Benoîte Groult remarca que El segundo sexo no surgió de una reivindicación militante y menos aún de cualquier deseo de revancha.

\footnotetext{
"Hasta ese momento, Beauvoir había logrado llevar una vida brillante y, por otro lado, en ese período turbio de la posguerra, los derechos de las mujeres estaban lejos de ser una cuestión prioritaria. El término "feminismo" no había sido pronunciado nunca, y en Francia no existía ninguna asociación para los derechos de las mujeres" (Groult, 2010: 30).
}

Revista Zona Franca- Centro de estudios interdisciplinario sobre las mujeres (CEIM)- Maestría poder y sociedad desde la problemática de género (MG), Rosario, Argentina. ISSN, 2545-6504 http://zonafranca.unr.edu.ar/index.php/ZonaFrancal Numero 27 (2019). 
Entonces no fue hasta mayo del 68 que al menos una parte de las mujeres de aquel movimiento, decepcionadas e incluso escandalizadas por el sexismo de sus amigos "revolucionarios", redescubrieron y revalorizaron El segundo sexo, cuya publicación había provocado primero violentas controversias y luego silencio, y se reagruparon haciendo de su realidad de mujeres el lugar de una afirmación y luego de una reivindicación colectiva.

Para la propia Beauvoir será la oportunidad de asumir en su vida la problemática que planteara el texto: "Las mujeres -salvo en ciertos congresos que siguen siendo manifestaciones abstractas- no dicen "nosotras"; los hombres dicen "las mujeres", y ellas retoman estas palabras para designarse a sí mismas, pero no se plantean auténticamente como sujeto" (Beauvoir, 1954, 1: 18) con la sugerencia de posibles salidas de la inmanencia: "la libertad se halla íntegra en cada cual. Sólo por el hecho de permanecer abstracta y vacía en la mujer, ésta podría asumirse auténticamente en la rebelión, único camino abierto a quienes no tienen la posibilidad de construir nada. Es preciso que nieguen los límites de su situación e intenten abrirse los caminos del porvenir. (...) Para la mujer no hay más salida que trabajar por su liberación. Esa liberación sólo podría ser colectiva..." (Beauvoir, 1954, 2: 457).

El "nosotras" auspiciado en su impactante ensayo toma carnalidad personal cuando a inicios de los 70 la filósofa se incorpora al incipiente Mouvement de libération des femmes (MLF) y disputa a la militancia marxista la explicación de la alienación humana que no podría reducirse a una única causa.

Desde esta asunción del «nosotras» Beauvoir redacta el Manifiesto de las 343 que se publica el 5 de abril de 1971 en Le Nouvel Observateur:

\footnotetext{
"Un millón de mujeres abortan cada año en Francia. Ellas lo hacen en condiciones peligrosas debido a la clandestinidad a la que son condenadas cuando esta operación, practicada bajo control médico, es una de las más simples. Se sume en el silencio a estos millones de mujeres. Yo declaro que soy una de ellas. Declaro haber abortado. Al
}

Revista Zona Franca- Centro de estudios interdisciplinario sobre las mujeres (CEIM)- Maestría poder y sociedad desde la problemática de género (MG), Rosario, Argentina. ISSN, 2545-6504 http://zonafranca.unr.edu.ar/index.php/ZonaFrancal Numero 27 (2019). 
igual que reclamamos el libre acceso a los medios anticonceptivos, reclamamos el aborto libre" (Suárez Briones, 2013: 15).

Este acontecimiento conocido y replicado desde el enunciado "Yo aborté" evidencia de modo contundente la colectivización del "yo" y su contribución a la producción de subjetividades sociales que señalara July Chaneton. La proclama "yo aborté" está sostenida por el "nosotras" al que evoca, del que emerge y que en genealogía con la actualidad deviene "nosotr*s"

En su situación Beauvoir escribe, reitero: "Yo declaro que soy una de ellas. Declaro haber abortado" y la afirmación impide objetivar a las mujeres en la distancia de la despersonalización. El "yo" del manifiesto es claramente una $x$ vacía que se plenifica con el nombre propio de cada firmante. Nombre propio del que carece La mujer rota, alienada en su inmanencia...

Pero Simone de Beauvoir puede poner en escena su nombre así como su cuerpo en las manifestaciones públicas colectivas. Lo hacía previamente a su militancia feminista; lo hace, asumiéndose en un nosotras. En este sentido Françoise Collin considera que Beauvoir no abandona la pretensión de excepción al seleccionar qué muestra de sí:

"es curioso que para afirmar públicamente su independencia, Simone de Beauvoir la haya inscripto con tanta obstinación dentro de la figura de la pareja heterosexual, aunque no fuera un matrimonio, ocultando las relaciones homosexuales en las que ella siempre jugó el papel de hermana mayor y de dominante" (Collin, 2010: 68).

De todas maneras, Beauvoir dedica un capítulo de El segundo sexo a "la lesbiana" que brinda un panorama de desasosiego y no invita precisamente a la identificación. Como señala Beatriz Suárez Briones, "es un capítulo deprimente para cualquier lesbiana, y lesbófobo en buena medida desde aquí y desde ahora"

I Sobre el carácter de tecnología de sí misma que tiene el lema "yo aborté" invito a leer Campagnoli (2005). Con la actualidad del "nosotr*s" me refiero a la apertura del sujeto de la gestación, de mujeres a personas gestantes, reconociendo la diversidad de identidades de género.

Revista Zona Franca- Centro de estudios interdisciplinario sobre las mujeres (CEIM)- Maestría poder y sociedad desde la problemática de género (MG), Rosario, Argentina. ISSN, 2545-6504 http://zonafranca.unr.edu.ar/index.php/ZonaFrancal Numero 27 (2019). 
(Suárez Briones, 2013: 17). Sin embargo, en el relato autobiográfico La fuerza de las cosas,

"Beauvoir cuenta que recibió multitud de cartas de agradecimiento y amor de mujeres y hombres homosexuales; también explicó que llamó a su libro el segundo sexo porque, dado que las y los homosexuales eran llamados el tercer sexo, las mujeres debían ser el segundo. Tuvieron que pasar más de 20 años parar que Simone de Beauvoir afirmara públicamente ser feminista" (Ibíd).

Al respecto, Alejandra Ciriza se expresa más atenta al contexto vital de la filósofa, al afirmar que

"en 1949 de Beauvoir se situaba en una posición compleja. No solo de la indecibilidad de su propia posición ante la institución de la heterosexualidad obligatoria, sino de su propia posición respecto de las mujeres, de su lugar en el mundo de la cultura. (...) A lo largo de su vida pudo ir matizándol[a], admitiendo y haciendo lugar público a su no heterosexualidad, (...), haciendo lugar a las otras en su vida, pero pudo hacerlo también a medida que el mundo se fue transformando y que las mujeres fuimos ingresando a otros mundos que excedían el destino de domesticidad y maternidad que nos había sido asignado como fatalidad única e inevitable" (Ciriza, 2012: 13 y 15).

\section{Aquelarre textual}

Recorrer el espacio biográfico de Simone de Beauvoir permite encontrar inflexiones de sentido en la producción del "yo", en tanto se aleja o se acerca al "nosotras", en cuanto coincide en esta enunciación, cuya modalidad paradigmática la constituye el Manifiesto de las 343.

Este aspecto de la escritura como tecnología de sí es tomado por otras analistas como un indicio de la singularidad filosófica de Beauvoir con un perfil propio aún antes de su encuentro con Jean-Paul Sartre. Es la lectura que realiza

Revista Zona Franca- Centro de estudios interdisciplinario sobre las mujeres (CEIM)- Maestría poder y sociedad desde la problemática de género (MG), Rosario, Argentina. ISSN, 2545-6504 http://zonafranca.unr.edu.ar/index.php/ZonaFrancal Numero 27 (2019). 
María Marta Herrera a partir de los hallazgos percibidos en los diarios de juventud de la filósofa:

"En 1990 Sylvie Le Bon de Beauvoir dona a la Biblioteca Nacional los cuadernos redactados por su madre adoptiva cuando era estudiante, en ese período. En el diario del año 1927 en el que Beauvoir concurre a la Sorbonne -y dos años antes de su encuentro con Sartre- Margaret Simons, que participó en su transcripción, encuentra cuatro puntos que invalidan la lectura tradicional de Beauvoir en relación a su dependencia sartreana" (Herrera, 2010: 23).

Este punteo puede sintetizarse en: su lucha contra la desesperación la ha llevado a la filosofía; el tema del conflicto entre sí y el otro es central y no derivado de otras teorizaciones; sus afirmaciones no serían mera ilustración del existencialismo sartreano pues los indicios de "no se nace mujer, se llega a serlo" estarían en estos diarios que contendrían, incluso, la noción de "mala fe".

De esta lectura rescatamos una cita del diario de 1927 en la que el yo se pone al servicio de la reflexión sobre la situación de "ser mujer":

"Pero yo querría tanto tener el derecho yo también de ser muy simple y muy débil, de ser una mujer; en qué mundo desierto camino, tan árido, con los únicos oasis de mi estima intermitente por mí misma (...) Yo cuento conmigo; yo sé que puedo contar conmigo. Pero desearía tanto no tener necesidad de contar conmigo" (Beauvoir en Herrera, 2010: 24).

Podemos advertir aquí una paradoja existencialista así como una conflictividad feminista, eso depende de qué mundos circundantes abramos en nuestra lectura. A lo mejor es esta necesidad de no tener que contar consigo la que la lleva a escribir en La fuerza de las cosas, volumen posterior de la autobiografía: "He querido que en este relato circule mi sangre; he querido arrojarme a él, todavía viva" (Beauvoir, 1964: 10). Arrojarse al relato como una bruja a la hoguera, ¿el gesto de una pura singularidad o de una consumación para

Revista Zona Franca- Centro de estudios interdisciplinario sobre las mujeres (CEIM)- Maestría poder y sociedad desde la problemática de género (MG), Rosario, Argentina. ISSN, 2545-6504 http://zonafranca.unr.edu.ar/index.php/ZonaFranca| Numero 27 (2019). 
el nosotras? Aun cuando aceptemos una respuesta dilemática, no podemos negar que "hay alguien ahí".

\section{Bibliografía}

ARFUCH, Leonor (2002) El espacio biográfico. Dilemas de la subjetividad contemporánea, Bs As: FCE.

BEAUVOIR, Simone de (1954 [1949]) El segundo sexo, Vol 1 y Vol 2, Bs. As: Psique.

BEAUVOIR, Simone de (1999 [1958]) Memorias de una joven formal. Bs. As: Sudamericana.

BEAUVOIR, Simone de (1964 [1963]) La fuerza de las cosas, Bs. As: Sudamericana.

BOURDIEU, Pierre y Roger CHARTIER (2011) "La lectura: una práctica cultural", en Pierre Bourdieu, El sentido social del gusto. Elementos para una sociología de la cultura, Bs. As: Siglo XXI Editores.

BUTLER, Judith (2001 [1990]) El género en disputa. El feminismo y la subversión de la identidad, Barcelona: Paidós.

CAMPAGNOLI, Mabel Alicia (2005) "El feminismo es un humanismo. La década de los 70 y 'lo personal es político'" en Andrea Andújar et al. (comp.) Historia, Género y Política en los 70, BsAs: Feminaria.

CATELLI, Nora (1991) El espacio autobiográfico, Barcelona: Lumen.

CHANETON, July (2007) Género, poder y discursos sociales, Bs. As: Eudeba.

CIRIZA, Alejandra (2012) "Simone de Beauvoir. De la fenomenología del cuerpo a la utopía de la libertad", Revista Nomadías Noviembre, Número 16, 11 21.

COLLIN, Françoise (2010) "No se nace mujer y se nace mujer. Las ambigüedades de Simone de Beauvoir" en Beatriz Cagnolati y María Luisa Femenías (comps.) Simone de Beauvoir. Las encrucijadas de "el otro sexo", La Plata: EdULP.

Revista Zona Franca- Centro de estudios interdisciplinario sobre las mujeres (CEIM)- Maestría poder y sociedad desde la problemática de género (MG), Rosario, Argentina. ISSN, 2545-6504 http://zonafranca.unr.edu.ar/index.php/ZonaFrancal Numero 27 (2019). 
GROULT, Benoîte (2010) "La lucha por las mujeres" en Beatriz Cagnolati y María Luisa Femenías (comps.) Simone de Beauvoir. Las encrucijadas de "el otro sexo", La Plata: EdULP.

GRAU, Olga y Alejandra CASTILLO (2012) "Somos herederas de Simone de Beauvoir. Entrevista con Alejandra Ciriza", Revista Nomadías, Julio, Número 15, 231-250.

HERRERA, María Marta (2010) "Simone de Beauvoir, filósofa: algunas consideraciones" en Beatriz Cagnolati y María Luisa Femenías (comps.) Simone de Beauvoir. Las encrucijadas de "el otro sexo". La Plata: EdULP.

LECIÑANA BLANCHARD, Mayra (2010) "Simone de Beauvoir: Nuevas aproximaciones a la (auto) construcción del sujeto mujer" en Beatriz Cagnolati y María Luisa Femenías (comps.) Simone de Beauvoir. Las encrucijadas de "el otro sexo". La Plata: EdULP.

LE DCEUFF, Michèle (1993) El estudio y la rueca. De las mujeres, de la filosofía, etc., Madrid: Cátedra.

LÉJEUNE, Philippe (1994) El pacto autobiográfico y otros estudios, Málaga: Megazul.

LÓPEZ PARDINA, Teresa (1999) "Simone de Beauvoir y Sartre: Coincidencias y diferencias" en Jornadas de Homenaje a Simone de Beauvoir en el Cincuentenario del Segundo Sexo. BsAs, FFyL, UBA, 5 y 6 de agosto.

LÓPEZ SÁENZ, María Carmen (2012) "M. Merleau-Ponty (1908-1961) y S. De Beauvoir (1908-1986). El cuerpo fenoménico desde el feminismo" en Sapere Aude v.3, n.6. Belo Horizonte: º sem. pp.182-199.

ONG, Walter (1987 [1982]) Oralidad y Escritura. Tecnologías de la palabra, México: FCE.

RICOEUR, Paul (1996) Sí mismo como otro, Madrid: Siglo XXI.

RORTY, Richard (1990 [1967]) El giro lingüístico, Barcelona: Paidós.

SUÁREZ BRIONES, Beatriz (2013) "Cuando las lesbianas éramos mujeres" en Beatriz Suárez Briones (ed.) Las lesbianas (no) somos mujeres. En torno a Monique Wittig, Barcelona: Icaria.

Revista Zona Franca- Centro de estudios interdisciplinario sobre las mujeres (CEIM)- Maestría poder y sociedad desde la problemática de género (MG), Rosario, Argentina. ISSN, 2545-6504 http://zonafranca.unr.edu.ar/index.php/ZonaFranca| Numero 27 (2019). 